\title{
IDENTIFYING THE MAGNETIC PERMEABILITY IN MULTI-FREQUENCY EM DATA INVERSION*
}

\author{
GIAN PIERO DEIDDA ${ }^{\dagger}$, PATRICIA DÍAZ DE ALBA ${ }^{\ddagger}$, AND GIUSEPPE RODRIGUEZ ${ }^{\ddagger}$
}

\begin{abstract}
Electromagnetic induction surveys are among the most popular techniques for non-destructive investigation of soil properties in order to detect the presence of either ground inhomogeneities or of particular substances. In this paper we develop a regularized algorithm for the inversion of a nonlinear mathematical model well established in applied geophysics, starting from noisy electromagnetic data collected by varying both the height of the measuring device with respect to the ground level and its operating frequency. Assuming the conductivity to be known in advance, we focus on the determination of the magnetic permeability of the soil with respect to depth, and give the analytical expression of the Jacobian matrix of the forward model, which is indispensable for the application of the inversion algorithm. Finally, numerical experiments on synthetic data sets illustrate the effectiveness of the method.
\end{abstract}

Key words. Regularization, nonlinear inverse problems, electromagnetic induction

AMS subject classifications. 65F22, 65H10, 65R32, 86A22

1. Introduction. Frequency domain electromagnetic induction (EMI) techniques are among the most used geophysical methods for near-surface characterization in a variety of domains, from environment surveying to precision agriculture, from archaeology to unexploded ordnance (UXO) detection [4, 12, 13, 14, 15, 18, 19, 20, 24, 26, 29]. The typical measuring instrument is composed by two coils at distance $\rho$, one transmitter and one receiver (loop-loop device), and produces a single frequency scanning signal. The dipoles may be aligned horizontally or vertically with respect to the ground level.

Traditionally employed as a mapping tool, today this technique is more and more used as an imaging tool thanks to the growing availability of ground-based multi-coil and multifrequency EM devices (e.g., Geonics MK-2, Geophex GEM-2, CMD mini-Explorer, CMD Explorer, Dualem-21S, and Dualem-421S), which provide comprehensive multi-depth data, able to quantify the subsoil variability. These devices produce simultaneous measurement of both the real and imaginary parts of the ratio between the secondary magnetic field and the primary magnetic field components, providing information about the amplitude and the phase of the signal. The real part, or the in-phase component, is mainly affected by the magnetic permeability of the subsoil; the imaginary part, also called the out-of-phase or quadrature component, mainly by the electrical conductivity.

During the last decades, much effort has been made to invert the out-of-phase data in order to retrieve a 1D layered model of electrical conductivity, using linear (see, e.g., [2, 5, 16]) or nonlinear models $[3,6,10,23]$ and assuming a fixed magnetic permeability equal to the free space value, i.e., $\mu_{0}=4 \pi 10^{-7} \mathrm{H} / \mathrm{m}$ (Henry/meter). In many cases, however, EMI data is equally affected by magnetic susceptibility and electrical conductivity.

The presence or absence of magnetically susceptible materials causes significant differences in the observed responses. Over a nonmagnetic earth model, the in-phase response goes to zero as the operating frequency decreases, while it becomes negative when susceptible targets are present with more negative values as the magnetic permeability increases; see Remark 3.1 below. The negative values of the in-phase response at low frequencies cannot

* Received February 15, 2017. Accepted June 12, 2017. Published online on July 7, 2017. Recommended by L. Reichel.

${ }^{\dagger}$ Department of Civil, Environmental, and Architectural Engineering, University of Cagliari, Piazza d'Armi 1, 09123 Cagliari, Italy (gpdeidda@unica.it).

${ }^{\ddagger}$ Department of Mathematics and Computer Science, University of Cagliari, Viale Merello 92, 09123 Cagliari, Italy (patricia.diazdealba@gmail.com, rodriguez@unica.it). 
be reproduced by a pure conductivity model. As pointed out in [8], an inversion algorithm that properly takes into account the effects of susceptibility is always required, whether or not the knowledge of the variation of the magnetic permeability within the earth is desired; see also [22, 30]. Indeed, since susceptibility influences data differently across the range of operating frequencies, inverting for just the conductivity can produce inaccurate solutions and unreliable conductivity models [31] as data strongly affected by susceptibility will be underfit while less-affected data will be overfit to compensate. In this situation, real features and artefacts in the inverted conductivity model are often indistinguishable.

Both linear and nonlinear models are severely ill-conditioned, and the error propagation must be controlled via regularization algorithms. An algorithm for the solution of a nonlinear model [10, 27, 28] is described in [6], where the computation is performed by a Gauss-Newton method, regularized by means of the truncated singular value decomposition (TSVD) [9]. The method proposed in the paper deals with the classical approach of neglecting the effects of magnetic permeability and recovers the conductivity from the quadrature component of the signal. The loop-loop device is assumed to use a single scanning frequency, and multiple measurements are obtained by varying the height of the instrument above the ground. A relaxation parameter ensures both the convergence of the iterative method and the positivity of the solution. The algorithm has been extended to deal with multi-frequency data in [7], where experiments are performed with both real and imaginary synthetic data sets.

In this work we fix our attention on the identification of the magnetic permeability. The main result is to obtain stable formulas for the computation of the Jacobian of the model with respect to the magnetic permeability variation, which is required in order to solve the problem using the Gauss-Newton iteration. We investigate numerically the conditioning of the problem, as well as the performance of the analytically exact Jacobian in comparison to its finite difference approximation, both in terms of accuracy and of computing time. Then, under the working assumption that the conductivity is known in advance, we verify the reliability of the inversion algorithm and investigate the information content of data sets obtained by varying either the scanning frequency of the device or its height above the ground. The use of computer generated data sets with a noise level typical of real applications allows us to analyze the behaviour of the algorithm in a controlled setting. This is an essential step, before facing the more challenging task of determining both the conductivity and the permeability from experimental data.

In Section 2 we describe the nonlinear forward model which expresses the EMI measurements typical of a loop-loop system as functions of the electromagnetic features of the soil. Section 3 deals with the inverse problem and the solution method. The analytical expression of the Jacobian of the forward model is given in Section 4. Section 5 discusses our numerical experiments, and Section 6 summarizes the content of the paper and describes our intentions for future work.

2. The forward problem. We briefly recall here the forward model which describes the data measured by a loop-loop EM device, when the distribution of the conductivity and the magnetic permeability in the subsoil layers is known. It is obtained from Maxwell's equations after introducing suitable simplifications to account for the symmetry of the problem. It has been described in [10] and [27, 28]. Each subsoil layer is assumed to have thickness $d_{k}$, to be characterized by a conductivity $\sigma_{k}$ and a magnetic permeability $\mu_{k}$, for $k=1, \ldots, n$; see Figure 2.1. The thickness $d_{n}$ of the deepest layer is considered infinite. The two coils of the measuring device are at height $h$ above the ground, and their distance is $\rho$.

Let

$$
u_{k}(\lambda)=\sqrt{\lambda^{2}+\mathrm{i} \sigma_{k} \mu_{k} \omega}, \quad k=1, \ldots, n,
$$




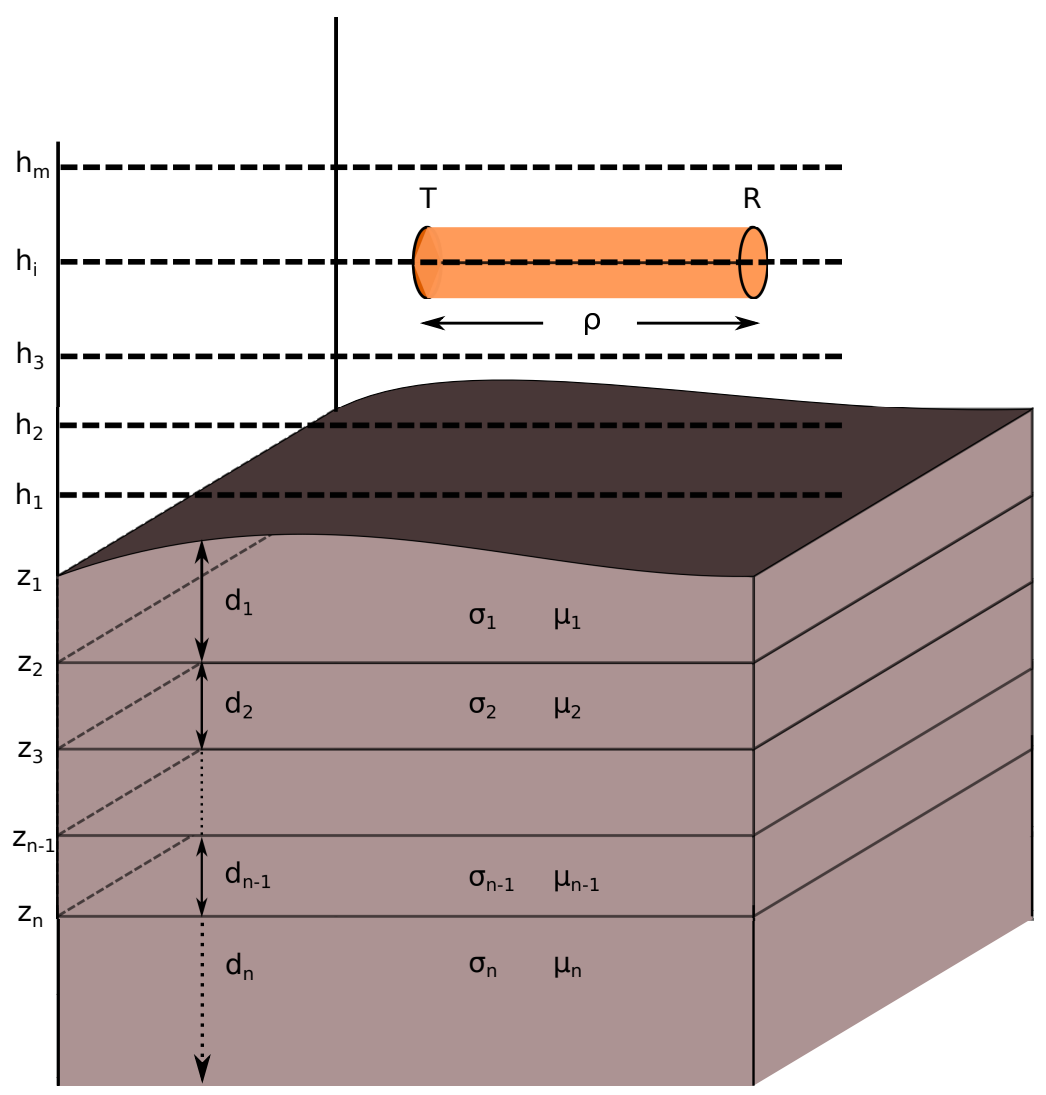

FIG. 2.1. Discretization and representation of the subsoil.

where $\omega$ is the angular frequency of the electromagnetic wave generated by the instrument, that is, $2 \pi$ times the frequency in Hertz, and $\mathrm{i}=\sqrt{-1}$. The variable $\lambda$ ranges from zero to infinity, and it measures the ratio between the depth below the ground surface and the inter-coil distance $\rho$. If we denote the characteristic admittance in the $k$-th layer by

$$
N_{k}(\lambda)=\frac{u_{k}(\lambda)}{\mathrm{i} \mu_{k} \omega}, \quad k=1, \ldots, n,
$$

then it is shown in [27] that the surface admittance $Y_{k}(\lambda)$ at the top of the same layer satisfies the recursion

$$
Y_{k}(\lambda)=N_{k}(\lambda) \frac{Y_{k+1}(\lambda)+N_{k}(\lambda) \tanh \left(d_{k} u_{k}(\lambda)\right)}{N_{k}(\lambda)+Y_{k+1}(\lambda) \tanh \left(d_{k} u_{k}(\lambda)\right)},
$$

for the backward index $k=n-1, \ldots, 1$. To initialize the recursion we set $Y_{n}(\lambda)=N_{n}(\lambda)$. Notice that both the characteristic and the surface admittances depend upon the frequency $\omega$ via the functions $u_{k}(\lambda)$.

Assuming that the instrument coils are vertically aligned, the ratio of the secondary to the primary field $[6,10]$ is given by

$$
M_{1}(\boldsymbol{\sigma}, \boldsymbol{\mu} ; h, \omega)=-\rho^{3} \int_{0}^{\infty} \lambda^{2} e^{-2 h \lambda} R_{\omega, 0}(\lambda) J_{0}(\rho \lambda) d \lambda,
$$




\section{ETNA}

Kent State University and

Johann Radon Institute (RICAM)

where $\boldsymbol{\sigma}=\left(\sigma_{1}, \ldots, \sigma_{n}\right)^{T}, \boldsymbol{\mu}=\left(\mu_{1}, \ldots, \mu_{n}\right)^{T}, J_{s}(\lambda)$ denotes the Bessel functions of the first kind of order $s$,

$$
R_{\omega, 0}(\lambda)=\frac{N_{0}(\lambda)-Y_{1}(\lambda)}{N_{0}(\lambda)+Y_{1}(\lambda)}
$$

$N_{0}(\lambda)=\lambda /\left(\mathrm{i} \mu_{0} \omega\right)$, and $Y_{1}(\lambda)$ is computed by the recursion (2.3). We have explicitly highlighted the dependence upon the frequency $\omega$ in $R_{\omega, 0}(\lambda)$ since it will be useful in the following.

For the horizontal orientation of the coils, (2.4) is replaced by

$$
M_{2}(\boldsymbol{\sigma}, \boldsymbol{\mu} ; h, \omega)=-\rho^{2} \int_{0}^{\infty} \lambda e^{-2 h \lambda} R_{\omega, 0}(\lambda) J_{1}(\rho \lambda) d \lambda .
$$

We remark that both (2.4) and (2.6) are complex-valued functions which can be expressed in terms of the Hankel transform

$$
\mathcal{H}_{\nu}[f](\rho)=\int_{0}^{\infty} f(\lambda) J_{\nu}(\rho \lambda) \lambda d \lambda, \quad \nu=0,1 .
$$

The available measuring devices, in general, return both the real (in-phase) and the imaginary (quadrature) components of the fields ratio.

3. The inverse problem. We assume the electrical conductivity to be known in advance for each of the $n$ subsoil layers. We will denote it by $\widehat{\sigma}=\left(\widehat{\sigma}_{1}, \ldots, \widehat{\sigma}_{n}\right)^{T}$. Then, the free variables are the values of the magnetic permeabilities in each layer, that is, the components of the vector $\boldsymbol{\mu} \in \mathbb{R}^{n}$.

Some recent EM devices allow for taking simultaneous measurements using a set of different operating frequencies. Moreover, the acquisition can be repeated at different heights. To account for this, we set $h=h_{1}, \ldots, h_{m_{h}}$ and $\omega=\omega_{1}, \ldots, \omega_{m_{\omega}}$ and consider the corresponding $2 m_{h} m_{\omega}$ data values $b_{i j}^{\nu}$, where $i=1, \ldots, m_{h}$ and $j=1, \ldots, m_{\omega}$. Here, $\nu=1,2$ represents the two possible orientations of the coils, vertical and horizontal.

The inverse problem consists of fitting the model to the data, that is, to determine the permeability vector $\boldsymbol{\mu}$ which produces the best approximation

$$
M_{\nu}\left(\widehat{\boldsymbol{\sigma}}, \boldsymbol{\mu} ; h_{i}, \omega_{j}\right) \approx b_{i j}^{\nu}, \quad \nu=1,2, i=1, \ldots, m_{h}, j=1, \ldots, m_{\omega} .
$$

Specifically, we vectorize the data values $b_{i j}^{\nu}$ in lexicographical order into $\mathbf{b} \in \mathbb{C}^{m}$ with $m=2 m_{h} m_{\omega}$. We proceed similarly for the model predictions, thus obtaining the vector $\mathbf{M}(\boldsymbol{\mu}) \in \mathbb{C}^{m}$, and minimize with respect to $\boldsymbol{\mu}$ the spectral norm of either the in-phase or the quadrature component of the residual between the data and the model, that is,

$$
\boldsymbol{\mu}^{*}=\arg \min _{\boldsymbol{\mu} \in \mathbb{R}^{n}} \frac{1}{2}\|\mathbf{r}(\boldsymbol{\mu})\|^{2},
$$

where $\mathbf{r}(\boldsymbol{\mu})=\operatorname{Re}(\mathbf{b}-\mathbf{M}(\boldsymbol{\mu}))$ or $\mathbf{r}(\boldsymbol{\mu})=\operatorname{Im}(\mathbf{b}-\mathbf{M}(\boldsymbol{\mu}))$. We will denote the components of the residual either by $r_{i}(\boldsymbol{\mu}), i=1, \ldots, m$, to emphasize their position in the vector $\mathbf{r}(\boldsymbol{\mu})$, or by $r_{i j}^{\nu}(\boldsymbol{\mu})$, when it is important to underline their dependence upon the height $h_{i}\left(i=1, \ldots, m_{h}\right)$, the frequency $\omega_{j}\left(j=1, \ldots, m_{\omega}\right)$, and the vertical or horizontal orientation $(\nu=1,2)$.

REMARK 3.1. Choosing to minimize either the in-phase or the quadrature component of the residual is in general a delicate decision. Figure 3.1 displays the graphs of the real and imaginary part of the device response (2.4) as a function of the operating frequency $f$, for different values of the relative permeability $\mu_{r}=\mu / \mu_{0}$; see [8]. The graphs show that 
either one of the two components can be more or less significant when different ranges of the frequency are considered. For example, for $f<10^{3} \mathrm{~Hz}$ or $f>10^{6} \mathrm{~Hz}$, the in-phase component is almost constant. Thus, the information content of the measurements is influenced by the electromagnetic features of the signal and consequently by the particular device being used and by the physical properties of the terrain. Moreover, since the quadrature component is not monotone, its minimization could lead to non-uniqueness of the solution. We also remark that negative values for the in-phase component are a clear indication of the presence of magnetic susceptible materials in the investigation area [8].
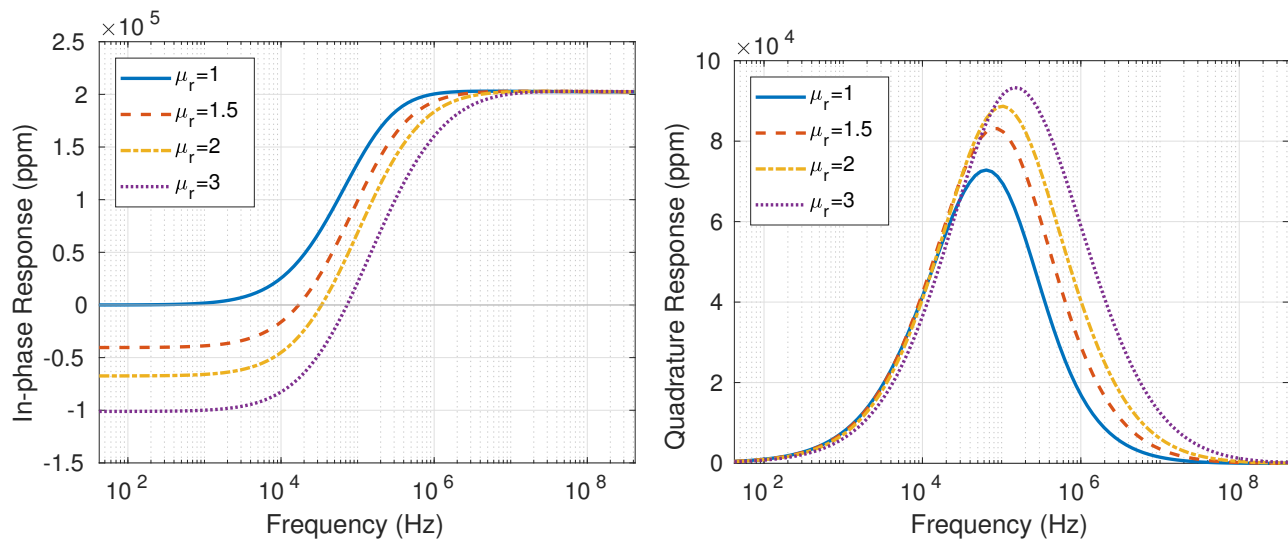

FIG. 3.1. In-phase (left) and quadrature (right) components of the response of an EM device, computed by (2.4), as a function of the operating frequency $f=\omega /(2 \pi)$. We let $\rho=1.66 \mathrm{~m}, h=1 \mathrm{~m}, \sigma=1 \mathrm{~S} / \mathrm{m}$ (Siemens $/$ meter), and $\mu_{r}=\mu / \mu_{0}=1,1.5,2,3$.

In general, a robust and reliable inversion algorithm would minimize both the real and the imaginary parts of the residual, possibly employing a tuning parameter to balance between the two. Given the difficulties due to nonlinearity and ill-conditioning, in order to fully understand the numerical issues involved in the computation, in this paper we chose to investigate the minimization of just one of the two components, leaving to future work the implementation of the full minimization of the complex residual and its application to experimental data.

Applying Newton's method to the solution of (3.1) would require the computation (or the approximation) of both the gradient vector and the Hessian matrix of the function to be minimized, leading to an algorithm characterized by a large computational complexity.

To overcome this difficulty, following [6], we resort to the Gauss-Newton method, which minimizes, at the $k$ th iteration step, the norm of a linear approximation of the residual $\mathbf{r}\left(\boldsymbol{\mu}_{k}+\mathbf{s}_{k}\right)$ with respect to the step size $\mathbf{s}_{k}$.

Let $\mathbf{r}(\boldsymbol{\mu})$ be Fréchet differentiable. We can write

$$
\mathbf{r}\left(\boldsymbol{\mu}_{k+1}\right) \simeq \mathbf{r}\left(\boldsymbol{\mu}_{k}\right)+J\left(\boldsymbol{\mu}_{k}\right) \mathbf{s}_{k},
$$

where $J(\boldsymbol{\mu})$ is the Jacobian of $\mathbf{r}(\boldsymbol{\mu})=\left(r_{1}(\boldsymbol{\mu}), \ldots, r_{m}(\boldsymbol{\mu})\right)^{T}$ defined by

$$
[J(\boldsymbol{\mu})]_{i j}=\frac{\partial r_{i}(\boldsymbol{\mu})}{\partial \mu_{j}}, \quad i=1, \ldots, m, j=1, \ldots, n .
$$

Here $n$ is again the number of the subsoil layers and $m=2 m_{h} m_{\omega}$. The analytical expression of the Jacobian matrix will be given in the next section. 


\section{ETNA}

Kent State University and

Johann Radon Institute (RICAM)

At each iteration $k$, the step length $\mathbf{s}_{k}$ is the solution of the linear least squares problem

$$
\min _{\mathbf{s} \in \mathbb{R}^{n}}\left\|\mathbf{r}\left(\boldsymbol{\mu}_{k}\right)+J_{k} \mathbf{s}\right\|
$$

with $J_{k}=J\left(\boldsymbol{\mu}_{k}\right)$ or some approximation to it, leading to the following iterative method

$$
\boldsymbol{\mu}_{k+1}=\boldsymbol{\mu}_{k}+\mathbf{s}_{k}=\boldsymbol{\mu}_{k}-J_{k}^{\dagger} \mathbf{r}\left(\boldsymbol{\mu}_{k}\right)
$$

The symbol $J_{k}^{\dagger}$ denotes the Moore-Penrose pseudoinverse of the matrix $J_{k}$ [1].

When the residuals $r_{i}\left(\boldsymbol{\mu}_{k}\right)$ are small or mildly nonlinear in a neighborhood of the solution, the Gauss-Newton method is expected to behave similarly to Newton's method [1]. We remark that, while the physical problem is obviously consistent, this is not necessarily true in our case, where the magnetic permeability $\mu(z)$ is approximated by a piecewise constant function and the data are noisy.

The damped Gauss-Newton method replaces the approximation (3.3) by

$$
\boldsymbol{\mu}_{k+1}=\boldsymbol{\mu}_{k}+\alpha_{k} \mathbf{s}_{k}
$$

where $\alpha_{k}$ is a relaxation parameter to be determined. To choose it, we use the ArmijoGoldstein principle [17]. This choice of $\alpha_{k}$ ensures convergence of the method provided that $\boldsymbol{\mu}_{k}$ is not a critical point [1]. The damped method allows us to include an important physical constraint in the inversion algorithm, i.e., the positivity of the solution. In our implementation, $\alpha_{k}$ is the largest step size in the sequence $2^{-i}, i=0,1, \ldots$, which both satisfies the Armijo-Goldstein principle

$$
\left\|\mathbf{r}\left(\boldsymbol{\mu}_{k}\right)\right\|^{2}-\left\|\mathbf{r}\left(\boldsymbol{\mu}_{k}+\alpha_{k} \mathbf{s}_{k}\right)\right\|^{2} \geq \frac{1}{2} \alpha_{k}\left\|J_{k} \mathbf{s}_{k}\right\|^{2}
$$

and ensures that all the components of the solution are positive.

Let us introduce the singular value decomposition (SVD) of the Jacobian matrix

$$
J_{k}=U \Gamma V^{T},
$$

where $\Gamma=\operatorname{diag}\left(\gamma_{1}, \ldots, \gamma_{p}\right)$, with $\gamma_{1} \geq \gamma_{2} \geq \cdots \geq \gamma_{p}>0, p$ is the rank of $J_{k}$, and $U, V$ are matrices with orthonormal columns $\mathbf{u}_{i}, \mathbf{v}_{i}$, respectively [1]. The condition number of a matrix can be defined in terms of the SVD as

$$
\operatorname{cond}\left(J_{k}\right)=\frac{\gamma_{1}}{\gamma_{p}}
$$

This quantity plays an important role in the accuracy of the solution of the least-squares problem (3.2), as it accounts for the propagation of the errors. Indeed the relative error in the solution of (3.2) is bounded by

$$
\frac{\operatorname{cond}\left(J_{k}\right)}{\eta \cos \theta}
$$

times the relative perturbation in $\mathbf{r}\left(\boldsymbol{\mu}_{k}\right)$, where $\eta=\frac{\left\|J_{k}\right\| \cdot\|\mathbf{s}\|}{\left\|J_{k} \mathbf{s}\right\|} \in\left[1, \operatorname{cond}\left(J_{k}\right)\right]$ and

$$
\theta=\arccos \frac{\left\|J_{k} \mathbf{s}\right\|}{\left\|\mathbf{r}\left(\boldsymbol{\mu}_{k}\right)\right\|} \in\left[0, \frac{\pi}{2}\right]
$$

is the angle between the right-hand side $\mathbf{r}\left(\boldsymbol{\mu}_{k}\right)$ and its projection $J_{k} \mathbf{s}$ on the range of $J_{k}$ [25, Theorem 18.1]. 
It is well known that the minimization of (3.1) is an extremely ill-conditioned problem. In particular, it has been observed in [6, Figure 2] that the Jacobian matrix $J$ of $\mathbf{r}$, expressed as a function of the conductivity vector $\sigma$, has a large condition number for any value of $\sigma$ in the solution domain. We will investigate the behaviour of the condition number with respect to the variation of the permeability $\boldsymbol{\mu}$ in Section 4 .

A common remedy to face ill-conditioning consists of replacing the least squares problem (3.2) by a nearby problem, whose solution is less sensitive to the error present in the data. This replacement is known as regularization.

A regularization method which particularly suits our problem, given the size of the matrices involved, is the truncated singular value decomposition (TSVD) [9]. The best rank $\ell$ approximation $\left(\ell \leq p=\operatorname{rank}\left(J_{k}\right)\right)$ to the Jacobian matrix, according to the spectral norm, can be obtained in terms of the SVD decomposition (3.5). This factorization allows us to replace the ill-conditioned Jacobian $J_{k}$ by a well-conditioned low-rank matrix $A_{\ell}$, such that

$$
\left\|J_{k}-A_{\ell}\right\|=\min _{\operatorname{rank}(A)=\ell}\left\|J_{k}-A\right\| .
$$

The matrix $A_{\ell}$ can be easily obtained by setting $A_{\ell}=U_{\ell} \Gamma_{\ell} V_{\ell}^{T}$, with

$$
\Gamma_{\ell}=\operatorname{diag}\left(\gamma_{1}, \ldots, \gamma_{\ell}\right), \quad U_{\ell}=\left[\mathbf{u}_{1}, \ldots, \mathbf{u}_{\ell}\right], \quad V_{\ell}=\left[\mathbf{v}_{1}, \ldots, \mathbf{v}_{\ell}\right] .
$$

Then, the regularized solution to (3.2) can be expressed as

$$
\mathbf{s}^{(\ell)}=-A_{\ell}^{\dagger} \mathbf{r}=-\sum_{i=1}^{\ell} \frac{\mathbf{u}_{i}^{T} \mathbf{r}}{\gamma_{i}} \mathbf{v}_{i},
$$

where $\mathbf{r}=\mathbf{r}\left(\boldsymbol{\mu}_{k}\right)$ and $\ell=1, \ldots, p$ is the regularization parameter.

When some kind of a priori information on the problem is available, e.g., the solution is a smooth function, it is sometimes useful to introduce a regularization matrix $L \in \mathbb{R}^{t \times n}$ $(t \leq n)$, whose kernel approximately contains the sought solution [9]. Under the assumption $\mathcal{N}\left(J_{k}\right) \cap \mathcal{N}(L)=\{0\}$, problem (3.2) is replaced by

$$
\min _{\mathbf{s} \in \mathcal{S}}\|L \mathbf{s}\|, \quad \mathcal{S}=\left\{\mathbf{s} \in \mathbb{R}^{n}: J_{k}^{T} J_{k} \mathbf{s}=-J_{k}^{T} \mathbf{r}\left(\boldsymbol{\mu}_{k}\right)\right\} .
$$

Very common choices for $L$ are the discretization of the first and second derivative operators, which we denote by $D_{1}$ and $D_{2}$, respectively.

The generalized singular value decomposition (GSVD) of the matrix pair $\left(J_{k}, L\right)$ is the factorization

$$
J_{k}=\tilde{U} \Sigma_{J} Z^{-1}, \quad L=\tilde{V} \Sigma_{L} Z^{-1},
$$

where $\Sigma_{J}$ and $\Sigma_{L}$ are diagonal matrices with nonnegative entries, $\tilde{U}$ and $\tilde{V}$ are matrices with orthonormal columns, and $Z$ is nonsingular. By the simultaneous factorization (3.6) it is possible to define a truncated GSVD (TGSVD) solution $\mathbf{s}_{\ell}$; see [9] for details.

Our algorithm for the regularized solution of (3.1) applies either TSVD or TGSVD to each step of the damped Gauss-Newton method (3.4). For a fixed value of the regularization parameter $\ell$, we substitute the truncated SVD or GSVD solution of (3.2) $\mathbf{S}^{(\ell)}$ to the step size $\mathbf{s}_{k}$ in (3.4), obtaining the following iterative method

$$
\boldsymbol{\mu}_{k+1}^{(\ell)}=\boldsymbol{\mu}_{k}^{(\ell)}+\alpha_{k} \mathbf{s}_{k}^{(\ell)} .
$$

We denote by $\boldsymbol{\mu}^{(\ell)}$ the solution at convergence. 
The choice of the regularization parameter $\ell$ is crucial in order to obtain a good approximation $\boldsymbol{\mu}^{(\ell)}$ of $\boldsymbol{\mu}$. In real applications, experimental data are affected by noise, so the data vector in the residual function (3.1) must be expressed as $\mathbf{b}=\widehat{\mathbf{b}}+\mathbf{e}$, where $\widehat{\mathbf{b}}$ contains the exact data and $\mathbf{e}$ is the noise vector. If the noise is Gaussian and an accurate estimate of $\|\mathbf{e}\|$ is available, then the discrepancy principle [9] determines $\ell$ as the smallest index such that

$$
\left\|\mathbf{b}-\mathbf{M}\left(\boldsymbol{\mu}^{(\ell)}\right)\right\| \leq \kappa\|\mathbf{e}\|,
$$

where $\kappa>1$ is a user-specified constant independent of $\|\mathbf{e}\|$.

In the absence of a trustworthy estimate of the noise level, many heuristic methods have been introduced to approximate a regularization parameter; see [21] for a review of methods and [11], where the parameter is estimated by comparing the solutions obtained by Tikhonov regularization and TSVD.

4. The Jacobian matrix of $r(\boldsymbol{\mu})$. As we saw in the previous section, being able to compute or approximate the Jacobian matrix $J(\boldsymbol{\mu})$ of $\mathbf{r}(\boldsymbol{\mu})$ is crucial for the implementation of an effective inversion algorithm.

In this section we give the explicit (i.e., analytical) expression of the Jacobian matrix. We will show numerically that the complexity of this computation is smaller than that required by a finite difference approximation. The following lemma is one of the main contributions of this work. In its statement we omit, for clarity, the variable $\lambda$.

LEMmA 4.1. The derivatives $Y_{k j}^{\prime}=\frac{\partial Y_{k}}{\partial \mu_{j}}, k, j=1, \ldots, n$, of the surface admittances (2.3) can be obtained starting from

$$
Y_{n n}^{\prime}=\frac{\sigma_{n}}{2 \mu_{n} u_{n}}-\frac{N_{n}}{\mu_{n}}, \quad Y_{n j}^{\prime}=0, \quad j=1, \ldots, n-1,
$$

and proceeding recursively for $k=n-1, n-2, \ldots, 1$ by

$$
\begin{aligned}
Y_{k j}^{\prime} & =N_{k}^{2} b_{k} Y_{k+1, j}^{\prime}, \quad j=n, n-1, \ldots, k+1, \\
Y_{k k}^{\prime} & =\frac{a_{k}-b_{k} N_{k} Y_{k+1}}{\mu_{k}^{2} \omega}\left(u_{k}-\frac{\sigma_{k}}{2 N_{k}}\right) \mathrm{i}+\frac{b_{k} d_{k} \sigma_{k}}{2 \mu_{k}}\left(N_{k}^{2}-Y_{k+1}^{2}\right), \\
Y_{k j}^{\prime} & =0, \quad j=k-1, k-2, \ldots, 1,
\end{aligned}
$$

where

$$
a_{k}=\frac{Y_{k+1}+N_{k} \tanh \left(d_{k} u_{k}\right)}{N_{k}+Y_{k+1} \tanh \left(d_{k} u_{k}\right)}, \quad b_{k}=\frac{1}{\left[N_{k}+Y_{k+1} \tanh \left(d_{k} u_{k}\right)\right]^{2} \cosh ^{2}\left(d_{k} u_{k}\right)} .
$$

Proof. From the definition of $u_{k}(\lambda)$ (2.1) and $N_{k}(\lambda)$ (2.2), we obtain

$$
\begin{aligned}
& \frac{\partial u_{k}}{\partial \mu_{j}}=\frac{\partial}{\partial \mu_{j}} \sqrt{\lambda^{2}+\mathrm{i} \sigma_{k} \mu_{k} \omega}=\frac{1}{2 N_{k}} \frac{\sigma_{k}}{\mu_{k}} \delta_{k j}, \\
& \frac{\partial N_{k}}{\partial \mu_{j}}=\frac{\partial}{\partial \mu_{j}} \frac{u_{k}}{\mathrm{i} \mu_{k} \omega}=\frac{u_{k}-\frac{\sigma_{k}}{2 N_{k}}}{\mu_{k}^{2} \omega} \mathrm{i} \delta_{k j},
\end{aligned}
$$

where $\delta_{k j}$ is the Kronecker delta, that is, 1 if $k=j$ and 0 otherwise. The recursion initialization (4.1) follows from $Y_{n}=N_{n}$; see Section 2. We have

$$
\begin{aligned}
Y_{k j}^{\prime}=\frac{\partial N_{k}}{\partial \mu_{j}} a_{k}+N_{k} \cdot \frac{\frac{\partial Y_{k+1}}{\partial \mu_{j}}+\frac{\partial N_{k}}{\partial \mu_{j}} \tanh \left(d_{k} u_{k}\right)+N_{k} \frac{\partial \tanh \left(d_{k} u_{k}\right)}{\partial \mu_{j}}}{N_{k}+Y_{k+1} \tanh \left(d_{k} u_{k}\right)} \\
-N_{k} a_{k} \cdot \frac{\frac{\partial N_{k}}{\partial \mu_{j}}+\frac{\partial Y_{k+1}}{\partial \mu_{j}} \tanh \left(d_{k} u_{k}\right)+Y_{k+1} \frac{\partial \tanh \left(d_{k} u_{k}\right)}{\partial \mu_{j}}}{N_{k}+Y_{k+1} \tanh \left(d_{k} u_{k}\right)},
\end{aligned}
$$


with $a_{k}$ defined as in (4.3). If $j \neq k$, then $\frac{\partial N_{k}}{\partial \mu_{j}}=\frac{\partial u_{k}}{\partial \mu_{j}}=0$, and we obtain

$$
Y_{k j}^{\prime}=N_{k}^{2} \frac{\frac{\partial Y_{k+1}}{\partial \mu_{j}}\left(1-\tanh ^{2}\left(d_{k} u_{k}\right)\right)}{\left[N_{k}+Y_{k+1} \tanh \left(d_{k} u_{k}\right)\right]^{2}}=N_{k}^{2} b_{k} Y_{k+1, j}^{\prime}
$$

The last formula with $b_{k}$ given by (4.3) avoids the cancellation in $1-\tanh ^{2}\left(d_{k} u_{k}\right)$.

If $j=k$, after some straightforward simplifications, we get

$$
\begin{aligned}
Y_{k k}^{\prime}=\frac{\partial N_{k}}{\partial \mu_{k}} & a_{k}+\frac{N_{k}}{N_{k}+Y_{k+1} \tanh \left(d_{k} u_{k}\right)}\left[Y_{k+1, k}^{\prime}\left(1-a_{k} \tanh \left(d_{k} u_{k}\right)\right)\right. \\
& \left.+\frac{\partial N_{k}}{\partial \mu_{k}}\left(\tanh \left(d_{k} u_{k}\right)-a_{k}\right)+\frac{d_{k}}{2} \frac{\sigma_{k}}{\mu_{k}}\left(1-a_{k} \frac{Y_{k+1}}{N_{k}}\right)\left(1-\tanh ^{2}\left(d_{k} u_{k}\right)\right)\right]
\end{aligned}
$$

This formula, using (4.3) and (4.4), leads to

$$
Y_{k k}^{\prime}=\frac{\partial N_{k}}{\partial \mu_{k}}\left(a_{k}-b_{k} N_{k} Y_{k+1}\right)+b_{k} \frac{d_{k}}{2} \frac{\sigma_{k}}{\mu_{k}}\left[N_{k}^{2}-Y_{k+1}^{2}\right] .
$$

The initialization (4.1) implies that $Y_{k j}^{\prime}=0$ for any $j<k$. In particular $Y_{k+1, k}^{\prime}=0$ and, since $N_{k} / u_{k}$ is constant, one obtains the expression of $Y_{k k}^{\prime}$ given in (4.2). This completes the proof.

THEOREM 4.2. The partial derivatives of the residual function $\mathbf{r}(\boldsymbol{\mu})$ are given by

$$
\frac{\partial r_{i j}^{\nu}(\boldsymbol{\mu})}{\partial \mu_{k}}= \begin{cases}\rho^{3} \mathcal{H}_{0}\left[\lambda e^{-2 h_{i} \lambda} \frac{\partial R_{\omega_{j}, 0}(\lambda)}{\partial \mu_{k}}\right](\rho), & \nu=1 \\ \rho^{2} \mathcal{H}_{1}\left[e^{-2 h_{i} \lambda} \frac{\partial R_{\omega_{j}, 0}(\lambda)}{\partial \mu_{k}}\right](\rho), & \nu=2\end{cases}
$$

for $i=1, \ldots, m_{h}, j=1, \ldots, m_{\omega}$, and $k=1, \ldots, n$. Here $\mathcal{H}_{\nu}(\nu=1,2)$ denotes the Hankel transform (2.7), $\rho$ is the inter-coil distance, $\frac{\partial R_{\omega, 0}(\lambda)}{\partial \mu_{k}}$ is the kth component of the gradient of the function (2.5), that is,

$$
\frac{\partial R_{\omega, 0}(\lambda)}{\partial \mu_{k}}=\frac{-2 \lambda \mathrm{i} \mu_{0} \omega}{\left(\lambda+\mathrm{i} \mu_{0} \omega Y_{1}(\lambda)\right)^{2}} \cdot \frac{\partial Y_{1}(\lambda)}{\partial \mu_{k}}
$$

and the partial derivatives $\frac{\partial Y_{1}(\lambda)}{\partial \mu_{k}}$ are given by Lemma 4.1.

Proof. The proof follows easily from Lemma 4.1 and from equations (2.4), (2.5), (2.6), and (3.1).

5. Numerical experiments. The inversion algorithm described in the previous sections has been implemented in the Matlab programming language. The numerical experiments shown in this section were performed on a dual Xeon CPU E5-2620 system (12 cores), running the Debian GNU/Linux operating system and Matlab 9.1.

In the first experiment, we investigate the ill-conditioning of the problem. We assume the loop-loop device is in the vertical orientation with a constant operating frequency $f=14600 \mathrm{~Hz}$ and an inter-coil distance $\rho=1 \mathrm{~m}$; these features are typical of one of the most widely used devices, the Geonics EM38. We let $n=m_{h}=10,20,30,40$, that is, we simulate a dataset with measurements detected at heights $h_{i}, i=1, \ldots, m_{h}$, starting from the ground level up to $1.9 \mathrm{~m}$, and we consider a discretization below the surface with the same number $n$ of layers until the depth of $3 \mathrm{~m}$. For each choice of $n$, we evaluate the Jacobian matrix $J$ 


\section{ETNA}

Kent State University and

Johann Radon Institute (RICAM)

at 100 random vectors in $\mathbb{R}^{n}$, with entries distributed in the interval $\left[\mu_{0}, \mu_{r} \mu_{0}\right]$, for a chosen value of $\mu_{r}$. For each example, we record the singular values of $J$, as computed by the svd function of Matlab. The scaling parameter $\mu_{r}$ represents the maximum value allowed for the relative permeability $\left(\mu_{r}=\mu / \mu_{0}\right)$, and it is initially set to 100 . The conductivity of the layers is fixed at a constant value.
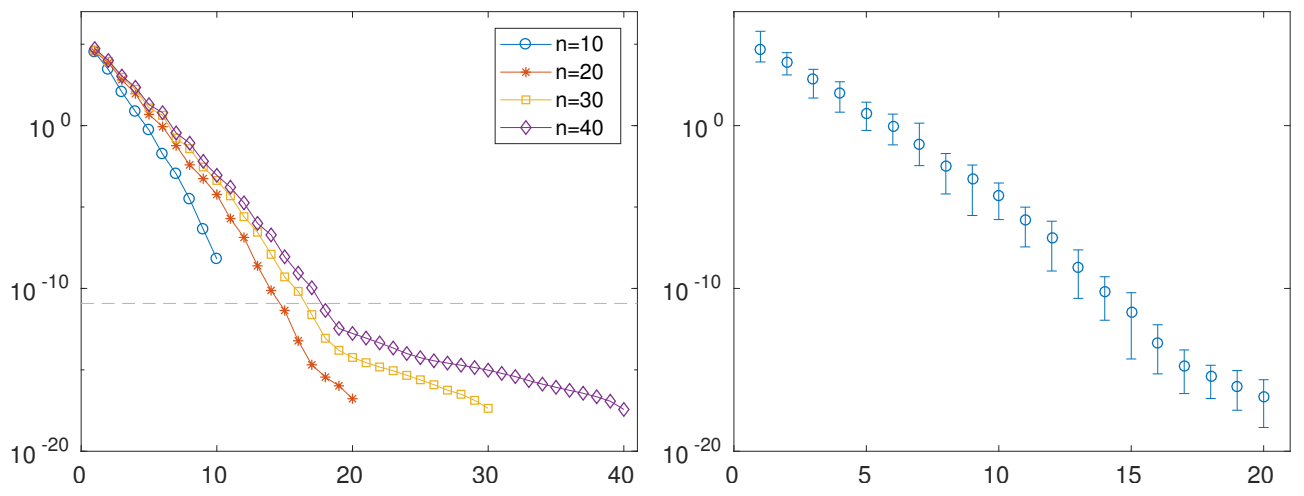

FIG. 5.1. Average of the singular values of the Jacobian $J(\boldsymbol{\mu})$ computed on 100 random points in $\mathbb{R}^{n}$, for $m_{h}=n=10,20,30,40$ (left-hand side); each component of $\boldsymbol{\mu}$ is in $\left[\mu_{0}, 100 \mu_{0}\right]$. The right-hand side graph shows the average singular values for $n=20$ together with their maximum and minimum value across the random tests.

The left-hand side graph in Figure 5.1 displays the average of the singular values for each choice of $n$; the dashed line marks the value of the product between the largest singular value and the machine epsilon $2.2 \cdot 10^{-16}$. It is immediate to observe that the singular values decay exponentially. The deviation from the initial decay rate, observed for $n>20$, is likely to be due to the error propagation caused by ill-conditioning, so it is reasonable to conjecture that the condition number increases with the size of the problem. The right-hand side graph in Figure 5.1 shows the average of the singular values for $n=20$ together with their maximum and minimum value across the 100 performed random tests. The graph shows that the variance from the average is small so that the Jacobian is uniformly ill-conditioned for permeabilities in $\left[\mu_{0}, \mu_{r} \mu_{0}\right]$ when $\mu_{r}=100$.

Figure 5.2 reports the average of the computed singular values when the maximum relative permeability $\mu_{r}$ takes the values $10,10^{2}, 10^{3}, 10^{4}$. The conditioning of the problem increases dramatically when the magnetic permeability is large, making it much harder to solve the inversion problem. This typically happens in the presence of ferromagnetic materials in the subsoil; consider that for iron $\mu_{r} \simeq 5 \cdot 10^{3}$. The fact that the problem is severely ill-conditioned also for small values of $\mu_{r}$ is in agreement with [6, Figure 2], where $\mu_{r}=1$ and the values of the conductivity are varied. Indeed, restoring ferromagnetic materials is a crucial issue in all inverse scattering problems.

Let us consider the following approximation of the partial derivatives of the residual components

$$
\frac{\partial r_{i}(\boldsymbol{\mu})}{\partial \mu_{j}} \simeq \frac{r_{i}\left(\boldsymbol{\mu}+\boldsymbol{\delta}_{j}\right)-r_{i}(\boldsymbol{\mu})}{\delta}, \quad i, j=1, \ldots, n,
$$

where $\boldsymbol{\delta}_{j}=\delta \mathbf{e}_{j}=(0, \ldots, 0, \delta, 0, \ldots, 0)^{T}$ and $\delta$ is a fixed constant. Resorting to a finite difference approximation of the Jacobian is a common approach in the solution of nonlinear 


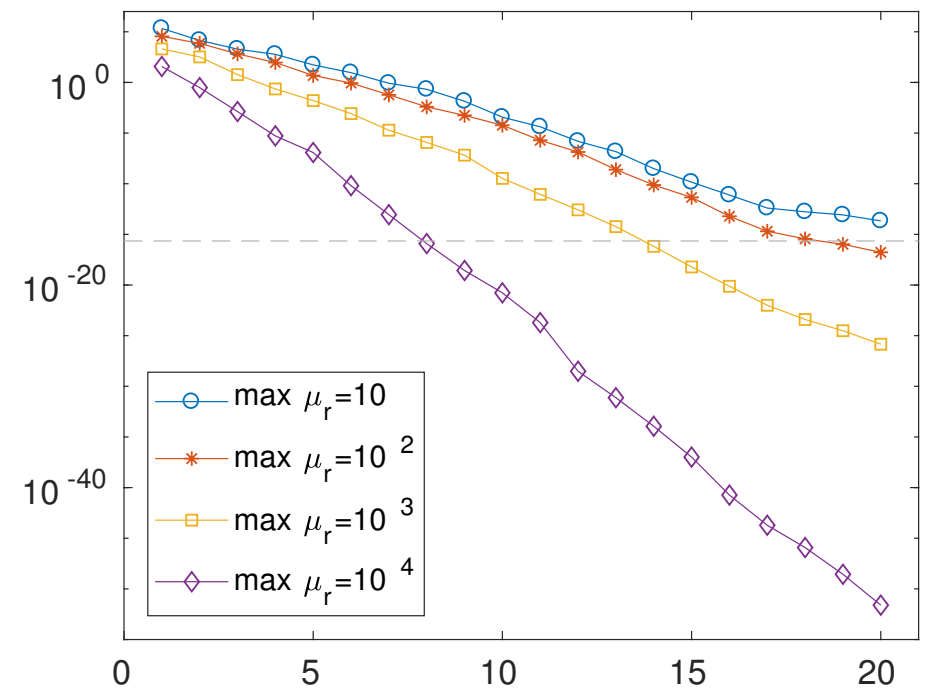

FIG. 5.2. Average of the singular values of the Jacobian $J(\boldsymbol{\mu})$ computed on 100 random points in $\mathbb{R}^{n}$, for $m=n=20$; each component of $\boldsymbol{\mu}$ is in $\left[\mu_{0}, \mu_{r} \mu_{0}\right]$, with $\mu_{r}=10,10^{2}, 10^{3}, 10^{4}$.
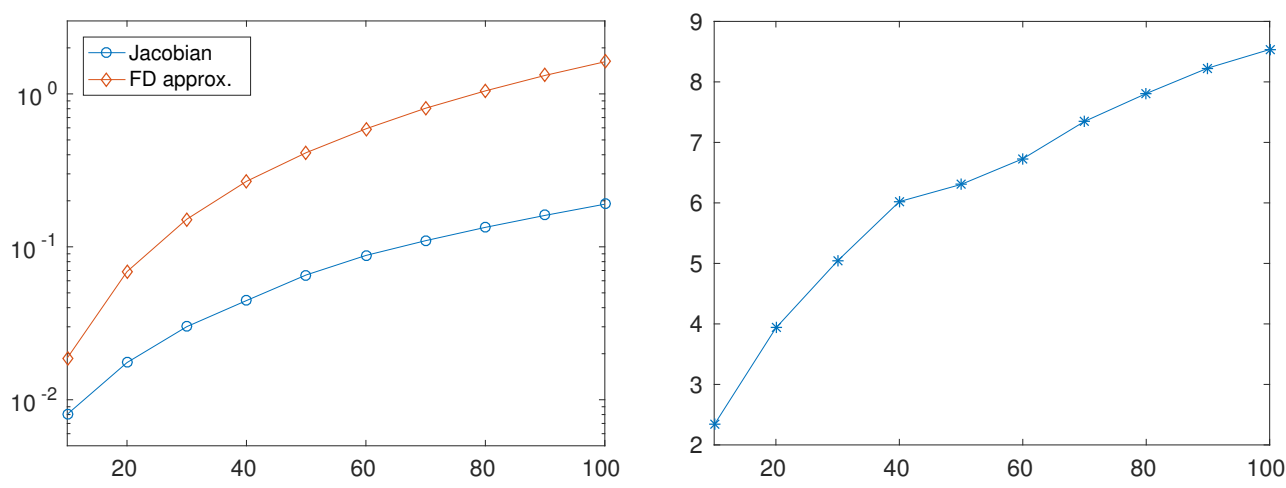

FIG. 5.3. Efficiency in the evaluation of the Jacobian $J$ of size $n=10,20, \ldots, 100$, compared to a finite difference approximation. The left-hand side graph displays the computing time in seconds for the two approaches; the right-hand side graph reports the speedup factor, i.e., the ratio between the timings of finite difference approximation and the analytically exact Jacobian.

problems; see, e.g., [10]. In [6] it was pointed out that the analytical computation of the Jacobian of the residual $\mathbf{r}(\boldsymbol{\sigma})$, expressed as a function of the conductivity vector $\boldsymbol{\sigma}$, has a smaller computational complexity than its finite difference approximation. This was a relevant observation since in many practical applications it is often assumed that the exact knowledge of the Jacobian is not strictly required, as its approximation leads to an algorithm with equivalent performance. The superiority in terms of complexity of the analytical evaluation of $J$ is confirmed also when the residual vector is seen as a function of $\boldsymbol{\mu}$. Figure 5.3 shows how the two approaches perform when the size of the problem increases. For $m_{h}=n=10,20, \ldots, 100$, we compute $J$ by the analytical formulas of Theorem 4.2 and by its finite difference approximation. The execution time is averaged over 100 repetitions of the computation. Figure 5.3 


\section{ETNA}

Kent State University and

Johann Radon Institute (RICAM)

reports the computing time for the two algorithms (left-hand side graph) together with the speedup factor (on the right), which we define as the ratio between the two timings. It is clear that the efficiency of the analytical computation increases with the size of the problem. Indeed, for $n=100$ the computation of the true Jacobian is almost 10 times faster than its finite difference approximation.
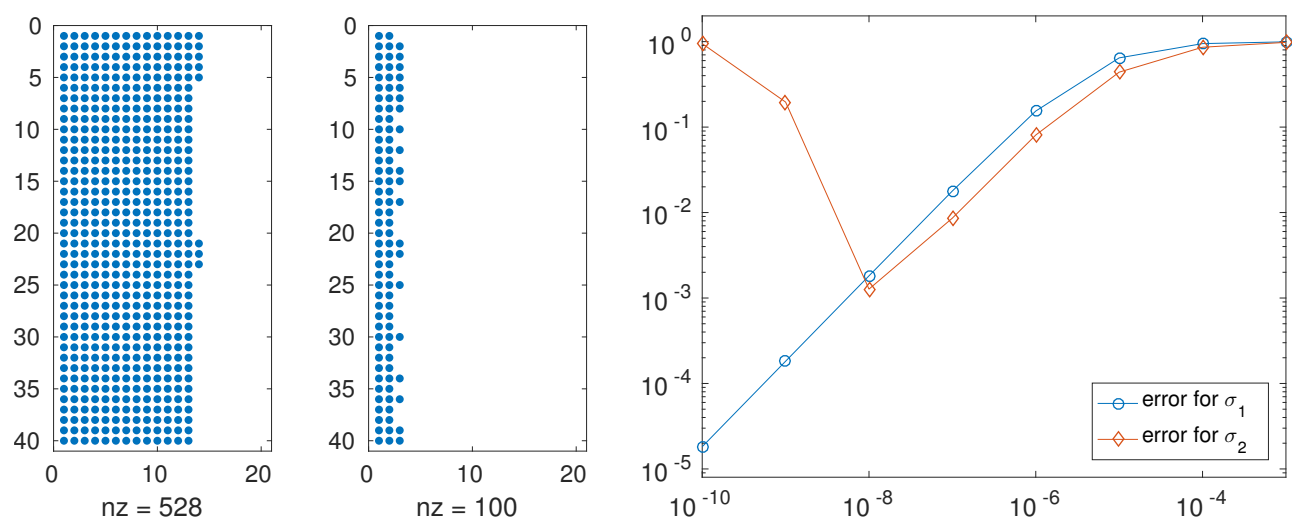

FIG. 5.4. The "spy plot" on the left shows the pattern of the nonzero elements for the Jacobian $J$ of size $40 \times 20$, as computed by the formulas in Section 4. The central graph displays the same information for $\widetilde{J}$, obtained by the approximation (5.1). The right-hand side plot represents the relative error for the first two singular values with respect to the variation of $\delta$ in (5.1).

The analytical representation of the Jacobian outperforms the finite difference approximation also from the point of view of accuracy, as the latter suffers from severe error propagation due to numerical cancellation. We let $\mu_{i} \in\left[\mu_{0}, \mu_{r} \mu_{0}\right], i=1, \ldots, n$, with $\mu_{r}=10$, and compare the Jacobian $J(\boldsymbol{\mu})$ of size $40 \times 20$ to its approximation $\widetilde{J}(\boldsymbol{\mu})$ obtained by (5.1) with $\delta=10^{-6}$. The two left-hand side graphs in Figure 5.4 display the pattern of the nonzero elements of $J$ and $\widetilde{J}$. The norm of the columns of $J$ decays very quickly, leading to vanishing entries starting from the 15 th column because of underflow. When we approximate the Jacobian by (5.1) for any $\delta \leq 10^{-3}$, cancellation makes the elements drop to zero already from the fourth columns; see the central graph in Figure 5.4. By computing the singular values of the two matrices using the svd function of Matlab, we can see that $\sigma_{i}(J)=0$ when $i \geq 12$ for the analytical Jacobian, while only the first three singular values of its finite difference approximation $\widetilde{J}$ are different from zero, denoting a dramatic loss of information.

The right-hand side graph in Figure 5.4 shows the relative differences

$$
\frac{\left|\sigma_{i}(J)-\sigma_{i}(\widetilde{J})\right|}{\sigma_{i}(J)}, \quad i=1,2,
$$

when $\delta$ in (5.1) takes the values $10^{-3}, 10^{-4}, \ldots, 10^{-10}$. While the approximation error for the first singular value decays monotonically, the error for the second one diverges when $\delta<10^{-8}$. The situation is even worse for the third singular value since $\sigma_{3}(\widetilde{J})$ grows from $1.2 \cdot 10^{-12}$ to $1.3 \cdot 10^{-5}$ when $\delta=10^{-3}, 10^{-4}, \ldots, 10^{-10}$, while $\sigma_{3}(J)=2.0 \cdot 10^{-20}$.

The accuracy of the finite difference approximation degrades in the presence of a larger magnetic permeability, as for $\mu_{i} \in\left[\mu_{0}, \mu_{r} \mu_{0}\right]$ with $\mu_{r} \geq 10^{2}$ only one column (and consequently one singular value) of $\widetilde{J}(\boldsymbol{\mu})$ is different from zero, while the analytical Jacobian preserves a larger number of nonzero columns. 
In order to illustrate the performance of the new formulas for the Jacobian in the inversion of EM data, we considered the following a priori model for the magnetic permeability as a function of depth

$$
\mu_{\theta}(z)=\mu_{0}\left(\theta \mathrm{e}^{-(z-1.2)^{2}}+1\right)
$$

where $\theta$ is a parameter to be chosen. The permeability takes values in $\left[\mu_{0},(\theta+1) \mu_{0}\right]$ and has a maximum at $z=1.2 \mathrm{~m}$. The conductivity of the subsoil is assumed to be known in advance and to be strongly correlated to the values of $\mu_{\theta}(z)$; we represent it by the model function $\sigma_{\theta}(z)=\theta \mathrm{e}^{-(z-1.2)^{2}}$ with values in $[0, \theta]$.

A synthetic data set is constructed by applying the forward model described in Section 2 to the sampling of $\mu_{\theta}(z)$ and $\sigma_{\theta}(z)$ at the depths $z_{i}=3.5(i-1) /(n-1), i=1, \ldots, n$. We initially fix $m_{h}=10, n=40$, and let the measurement heights be $h_{i}=1.9 i / m_{h}$, $i=1, \ldots, m_{h}$. We assume one operating frequency $f=14600 \mathrm{~Hz}$ for the instrument $\left(m_{\omega}=1\right)$ and both the horizontal and the vertical orientations. The data set is contaminated by additive Gaussian white noise with mean value zero and standard deviation $\tau / \sqrt{n}$. Since the noise level is known, we can estimate the regularization parameter $\ell$ in (3.7) by the discrepancy principle (3.8), where we set $\|\mathbf{e}\|=\tau=10^{-3}$ and $\kappa=1.5$.
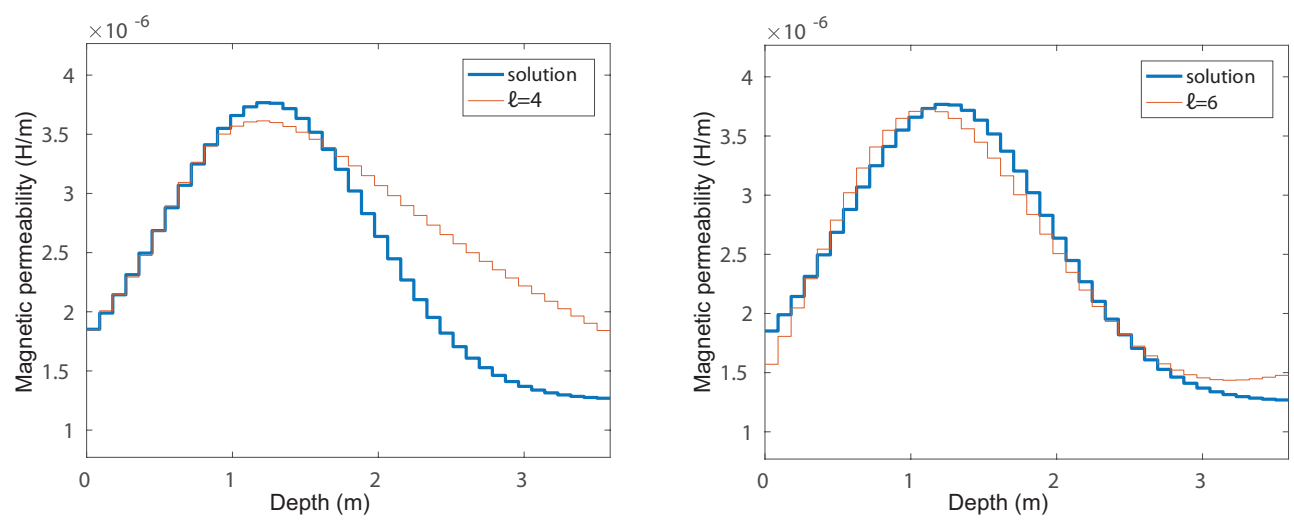

FIG. 5.5. Regularized solution obtained by fixing $\theta=2, m_{h}=10, m_{\omega}=1, n=40$, and $\tau=10^{-3}$. On the left, the result obtained by inverting the in-phase component of the data; on the right, the solution corresponding to the quadrature component.

Figure 5.5 shows the model solution compared to the approximated solutions obtained by applying our inversion algorithm either to the real part or to the imaginary part of the data values. We fixed $\theta=2$ corresponding to a moderate variation of the magnetic permeability. The solutions identified by the discrepancy principle lead both to a good global reconstruction of the test function and a reasonably accurate localization of its maximum.

Figure 5.6 displays the data set and the measurement predicted by the model for both the regularized solutions. A good match between the measured and the predicted data values guarantees a small residual. This is, in general, an indication that the chosen initial solution converged to an accurate local solution and that the algorithm was successful. Indeed, given the nonlinearity of the problem, the presence of a unique global minimum is not guaranteed. In these experiments we chose $\boldsymbol{\mu}^{(0)}=2 \mu_{0} \mathbf{u}$ with $\mathbf{u}=(1, \ldots, 1)^{T}$. Regarding the possible lack of uniqueness for the solution, see also Remark 3.1. 


\section{ETNA}

Kent State University and

Johann Radon Institute (RICAM)
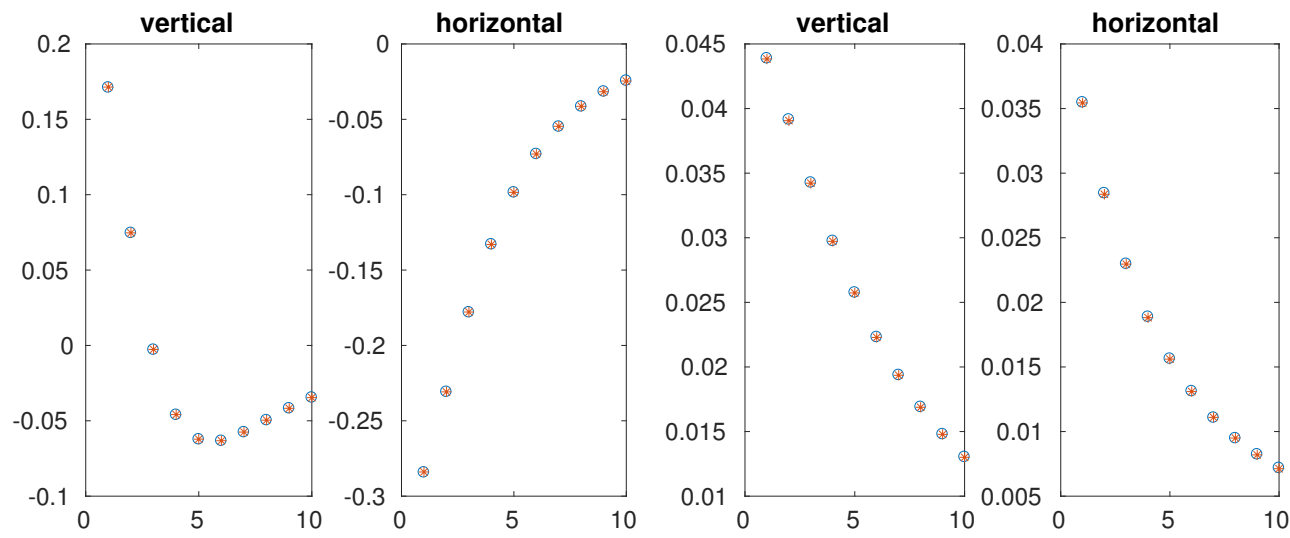

FIG. 5.6. Measured (circles) and predicted (asterisks) data values for the two solutions displayed in Figure 5.5; we display the data for both the vertical and the horizontal orientation of the device. The graphs on the left are obtained by inverting the in-phase component of the measured signal; the graphs on the right correspond to the quadrature component.
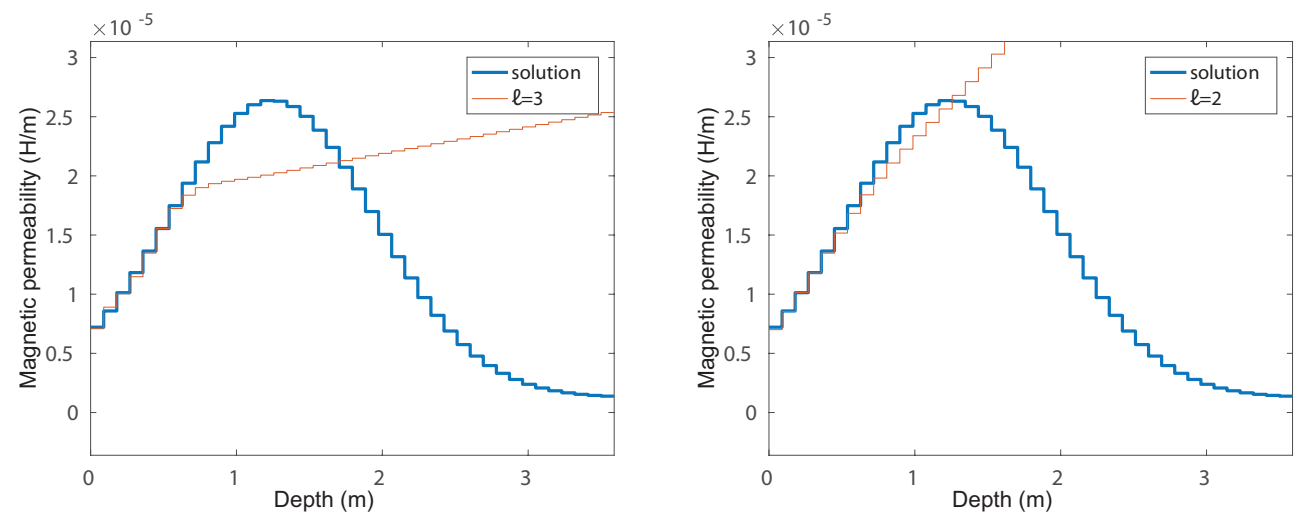

FIG. 5.7. Regularized solution obtained by fixing $\theta=20, m_{h}=20, m_{\omega}=1, n=40$, and $\tau=10^{-3}$. On the left, the result obtained by inverting the in-phase component of the data; on the right, the solution corresponding to the quadrature component.

The graphs in Figure 5.7 show the solutions obtained by fixing $\theta=20$, that is, assuming a wider variation of the magnetic permeability. All the other parameters are unchanged except $m_{h}=20$. The solutions displayed in the graphs are obtained by selecting the initial solution which produces the most significant converged solution, that is, $\boldsymbol{\mu}^{(0)}=8 \mu_{0} \mathbf{u}$ for the in-phase component inversion and $\boldsymbol{\mu}^{(0)}=10 \mu_{0} \mathbf{u}$ for the quadrature component inversion. Neither experiment correctly reproduces the behaviour of the solution: despite the size of the data is doubled, the algorithm is only able to detect the shape of the solution up to the depth of about 1 meter. We remark that the quality of the approximations does not improve by overestimating the value of the regularization parameter indicated by the discrepancy principle.

Varying the instrument height, that is, considering many $h_{i}$ values, has been the standard approach with first-generation devices, in order to obtain multiple data for each spatial point. This redundant information is required whenever one pretends to reconstruct the distribu- 


\section{ETNA}

Kent State University and

Johann Radon Institute (RICAM)
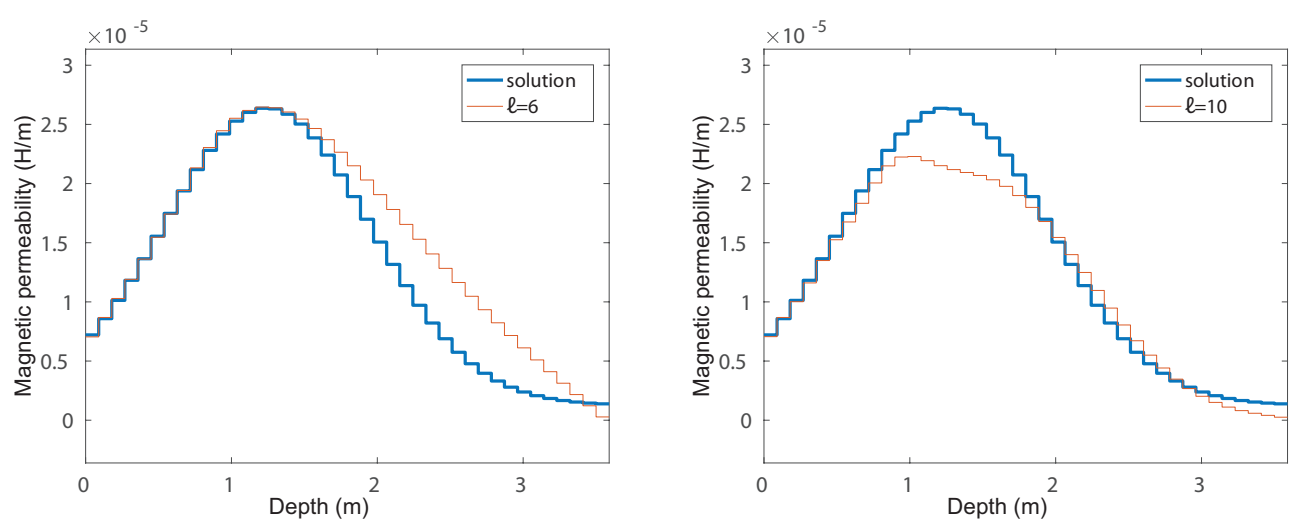

FIG. 5.8. Regularized solution obtained by fixing $\theta=20, m_{h}=1, m_{\omega}=6, n=40$, and $\tau=10^{-3}$. On the left, the result obtained by inverting the in-phase component of the data; on the right, the solution corresponding to the quadrature component.

tion of the electromagnetic features of the subsoil with respect to depth. Last-generation instruments, however, are either endowed with more than two coils, allowing for multiple values of the inter-coil distance $\rho$, or are able to perform simultaneous measurements with different angular frequencies $\omega_{j}=2 \pi f_{j}$. The Geophex GEM-2 falls into the last class of devices. It is endowed with two coils at a distance $\rho=1.66 \mathrm{~m}$, and it can be configured to use up to 10 frequencies for each data acquisition. In the following experiment we assume that each measurement is performed at 6 different frequencies, namely $f=775 \mathrm{~Hz}, 1175 \mathrm{~Hz}, 3925 \mathrm{~Hz}, 9825 \mathrm{~Hz}, 21725 \mathrm{~Hz}, 47025 \mathrm{~Hz}$, with the instrument at the height of $1 \mathrm{~m}$ and using both orientations. This means that we fix $m_{h}=1, m_{\omega}=6, \nu=1,2$, and apply the inversion algorithm with $m=12$ data values. This approach is known as "multi-frequency" (many frequencies) and "multi-view" (many receiving heights). The noise level is the same as before, i.e., $\tau=10^{-3}$, and the regularization parameter is chosen by the discrepancy principle (3.8) with $\kappa=1.5$.

We report in Figure 5.8 the results obtained by assuming the same strong variation of the permeability $(\theta=20)$ of the preceding experiment. The two graphs are computed by inverting either the in-phase or the quadrature component of the signal. The accuracy of the reconstructions demonstrates that varying the operating frequency of the device produces a data set containing much richer information than varying its height and suggests that this approach should be preferred in practical EM data inversion.

6. Conclusions and future developments. In this paper, we extend an inversion algorithm developed in [6] in order to deal with multi-frequency data as well as with multi-height data. We consider the problem of determining the magnetic permeability in the subsoil with respect to depth, assuming the electrical conductivity to be known. Moreover, we give analytical formulas for the Jacobian of the forward model, where the partial derivatives are computed with respect to the value of the permeability inside each ground layer.

The numerical experiments show that the analytically exact Jacobian is far more accurate than the finite difference approximation and faster to compute. The computation of the magnetic permeability appears to be harder than the evaluation of the conductivity, but the multi-frequency approach leads to accurate approximations of the solution when the algorithm is applied to noisy synthetic data; there are no significant differences when either the in-phase or the quadrature component of the data is considered. 


\section{ETNA}

Kent State University and

Johann Radon Institute (RICAM)

The results obtained, as well as the argumentation stated in Remark 3.1, suggest that the algorithm should be further developed in order to process both the real and imaginary parts of the measured signal, determining simultaneously the electrical conductivity and the magnetic permeability of the subsoil layers. The new computational procedure should be constructed with care, given the nonlinearity and the severe ill-conditioning of the forward model, and its performance should be tested on both synthetic ad experimental data sets. This work will be the subject of a future paper.

Acknowledgement. The authors wish to thank the reviewers for carefully reading the paper and for their comments, which lead to improvements of the presentation. The work of the authors was partially supported by Regione Sardegna grant CRP-78576 and INdAM-GNCS.

\section{REFERENCES}

[1] ̊̊. BJÖRCK, Numerical Methods for Least Squares Problems, SIAM, Philadelphia, 1996.

[2] B. BORCHERS, T. URAM, AND J. M. H. HENDRICKX, Tikhonov regularization of electrical conductivity depth profiles in field soils, Soil Sci. Soc. Am. J., 61 (1997), pp. 1004-1009. Package LINEM38 available at http://infohost.nmt.edu/ borchers/linem38.html.

[3] T. R. Brosten, F. D. Day-Lewis, G. M. Schultz, G. P. Curtis, and J. W. Lane, JR, Inversion of multi-frequency electromagnetic induction data for $3 D$ characterization of hydraulic conductivity, J. Appl. Geophys., 73 (2011), pp. 323-335.

[4] G. Cassiani, N. Ursino, R. Deiana, G. Vignoli, J. Boaga, M. Rossi, M. T. Perri, M. Blaschek, R. Duttmann, S. Meyer, R. Ludwig, A. Soddu, P. Dietrich, And U. Werban, Noninvasive monitoring of soil static characteristics and dynamic states: a case study highlighting vegetation effects on agricultural land, Vadose Zone J., 11 (2012), (16 pages).

[5] G. P. Deidda, E. Bonomi, AND C. MANZI, Inversion of electrical conductivity data with Tikhonov regularization approach: some considerations, Ann. Geophys., 46 (2003), pp. 549-558.

[6] G. P. DEIDDA, C. FENU, AND G. RodRIGUEZ, Regularized solution of a nonlinear problem in electromagnetic sounding, Inverse Problems, 30 (2014), Art. 125014 (27 pages).

[7] P. DíAZ DE AlBA AND G. RodRIGUEZ, Regularized inversion of multi-frequency EM data in geophysical applications, in Trends in Differential Equations and Applications, F. Ortegón Gallego, M. Redondo Neble, and J. Rodríguez Galván, eds., SEMA SIMAI Springer Series, 8, Springer, Cham, 2016, pp. 357-369.

[8] C. G. Farquharson, D. W. Oldenburg, And P. S. Routh, Simultaneous 1D inversion of loop-loop electromagnetic data for magnetic susceptibility and electrical conductivity, Geophysics, 68 (2003), pp. 1857-1869.

[9] P. C. HANSEN, Rank-Deficient and Discrete Ill-Posed Problems, SIAM, Philadelphia, 1998.

[10] J. M. H. Hendrickx, B. Borchers, D. L. Corwin, S. M. Lesch, A. C. Hilgendorf, and J. Schlue, Inversion of soil conductivity profiles from electromagnetic induction measurements, Soil Sci. Soc. Am. J., 66 (2002), pp. 673-685. Package NONLINEM38 available at http: // infohost.nmt.edu/ uorchers/nonlinem38.html.

[11] M. E. Hochstenbach, L. ReICHel, AND G. RodRIGUeZ, Regularization parameter determination for discrete ill-posed problems, J. Comput. Appl. Math., 273 (2015), pp. 132-149.

[12] H. HUANG AND I. J. Won, Automated anomaly picking from broadband electromagnetic data in an unexploded ordnance (UXO) survey, Geophysics, 68 (2003), pp. 1870-1876.

[13] E. Lascano, P. MARTinelli, AND A. Osella, EMI data from an archaeological resistive target revisited, Near Surf. Geophys., 4 (2006), pp. 395-400.

[14] N. Mansoor, L. SLATER, F. ARTigas, AND E. AuKEn, High-resolution geophysical characterization of shallow-water wetlands, Geophysics, 71 (2006), pp. B101-B109.

[15] P. MartinelLi AND M. C. DuPLAÁ, Laterally filtered $1 D$ inversions of small-loop, frequency-domain EMI data from a chemical waste site, Geophysics, 73 (2008), pp. F143-F149.

[16] J. D. MCNEILL, Electromagnetic terrain conductivity measurement at low induction numbers, Tech. Report TN-6, Geonics Limited, Mississauga, Canada, 1980.

[17] J. M. ORTEGA AND W. C. RheINBOLDT, Iterative Solution of Nonlinear Equations in Several Variables, Academic Press, New York, 1970.

[18] A. Osella, M. D. L. Vega, AND E. LasCANo, 3D electrical imaging of an archaeological site using electrical and electromagnetic methods, Geophysics, 70 (2005), pp. G101-G107.

[19] L. R. PASION, S. D. BILLINGS, AND D. W. OLDENBURG, Evaluating the effects of magnetic susceptibility in UXO discrimination problems, in Symposium on the Application of Geophysics to Engineering and Environmental Problems 2002, Society of Exploration Geophysicists, Tulsa, 2002, pp. 12UXO1-12UXO1. 
[20] L. PELlerin, Applications of electrical and electromagnetic methods for environmental and geotechnical investigations, Surv. Geophys., 23 (2002), pp. 101-132.

[21] L. REICHEL AND G. RODRIGUEZ, Old and new parameter choice rules for discrete ill-posed problems, Numer. Algorithms, 63 (2013), pp. 65-87.

[22] Y. SASAKI, J.-H. KIM, AND S.-J. CHO, Multidimensional inversion of loop-loop frequency-domain EM data for resistivity and magnetic susceptibility, Geophysics, 75 (2010), pp. F213-F223.

[23] G. SCHUltz AND C. RuPPEL, Inversion of inductive electromagnetic data in highly conductive terrains, Geophysics, 70 (2005), pp. G16-G28.

[24] D. Simpson, M. Van Meirvenne, T. Saey, H. Vermeersch, J. Bourgeois, A. Lehouck, L. CockX, AND U. W. VITHARANA, Evaluating the multiple coil configurations of the EM38DD and DUALEM-21S sensors to detect archaeological anomalies, Archaeol. Prospect., 16 (2009), pp. 91-102.

[25] L. TRefethen And D. BAU III, Numerical Linear Algebra, SIAM, Philadelphia, 1997.

[26] M. Viganotti, R. JACKSOn, H. KRAHn, AND M. DYER, Geometric and frequency EMI sounding of estuarine earthen flood defence embankments in Ireland using $1 D$ inversion models, J. Appl. Geophys., 92 (2013), pp. 110-120.

[27] J. R. WAIT, Geo-Electromagnetism, Academic Press, New York, 1982.

[28] S. H. WARD AND G. W. HOHMANN, Electromagnetic theory for geophysical applications, in Electromagnetic Methods in Applied Geophysics. Volume 1: Theory, M. N. Nabighian, ed., Investigation in Geophysics, 3 , Society of Exploration Geophysicists, Tulsa, 1987, pp. 131-311.

[29] R. YAO AND J. YANG, Quantitative evaluation of soil salinity and its spatial distribution using electromagnetic induction method, Agric. Water Manag., 97 (2010), pp. 1961-1970.

[30] M.-J. YI AND Y. SASAKI, 2-D and 3-D joint inversion of loop-loop electromagnetic and electrical data for resistivity and magnetic susceptibility, Geophys. J. Int., 203 (2015), pp. 1085-1095.

[31] Z. Zhang AND D. W. OldenBURG, Simultaneous reconstruction of 1-D susceptibility and conductivity from electromagnetic data, Geophysics, 64 (1999), pp. 33-47. 